\title{
Acute undifferentiated leukemia with myelofibrosis - A case report
}

\author{
Roopa Urs A.N ${ }^{1, *}$, Bhavani $K^{2}$
}

\begin{abstract}
${ }^{1}$ Department of Pathology, PKDAS Institute of Medical

Sciences,

Vaniamkulam, Palakaddu,

Kerala 682522.

${ }^{2}$ Department of Pathology,

Mahatma Gandhi Medical

College and Research Institute,

Sri Balaji Vidyapeeth,

(Deemed to be University),

Pillaiyarkuppam,

Puducherry-607402.
\end{abstract}

For Correspondence

${ }^{*}$ Roopa Urs AN,

Email:roopaurspr@gmail.com

Date of

Submisssion: 10-02-2018

Acceptance: 07-09-2018
Access this article online

Quick Response Code

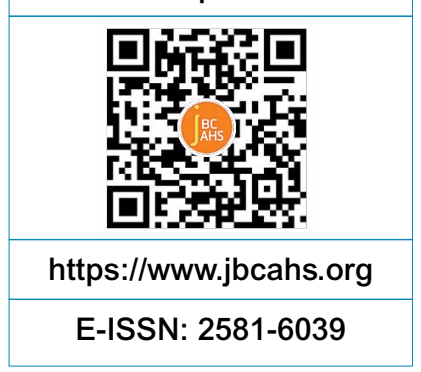

\section{ABSTRACT}

Acute undifferentiated leukemias (AUL) is the diagnosis of exclusion and is very rare, the morphology of leukemic cells is not specific. Here we report a rare case of AUL with myelofibrosis with an unusual morphology. A 40 year female presented with anaemia and hepatosple enomegaly with pancytopenia and leucoerythroblastic blood picture on peripheral blood smear underwent bone marrow studies. Bone marrow biopsy showed infiltration of blasts with scant cytoplasm, round to irregular nucleus, dispersed chromatin with grade 3 fibrosis by Reticulin stain and negative Masson trichrome stain. Immunohistochemistry (IHC) on bone marrow biopsy showed CD 34 positivity and negative for Tdt, MPO, CD3, CD20, CD61 markers. Thus diagnosed as Acute undifferentiated leukemia with myelofibrosis. AUL is the diagnosis of exclusion and before categorizing leukemia as undifferentiated, it is necessary to perform immunophenotyping with a comprehensive panel of monoclonal antibodies.

\section{Keywords}

Pancytopenia, hepatosplenomegaly, Immunohistochemistry

\section{INTRODUCTION}

Acute leukemia are clonal expansion of immature myeloid or lymphoid cells (blasts) in bone marrow, peripheral blood and other organs. The diagnosis and classification of acute leukemias rely on morphology, immunophenotyping with the expression of surface or cytoplasmic antigens, chromosomal abnormalities, and specific molecular genetic analyses. ${ }^{1}$

The 2008 edition of the World Health Organization Classification of Tumors of Hematopoietic and Lymphoid Tissues has described a special category called acute leukemias of ambiguous lineage', in which the AULs has been subcategorized. Here the leukemic cells express no more than one membrane marker of any given lineage. ${ }^{2}$ Acute undifferentiated leukemia is a rare subset of acute leukemias unclassifiable by the FrenchAmerican-British classification, where the lineage origin of leukemic blasts cannot be established by the associations of morphology, cytochemistry, and immunophenotyping with an extensive panel of monoclonal antibodies. ${ }^{3-5}$

AUL is the diagnosis of exclusion and is very rare, the morphology of leukemic cells is not specific. Here we report a rare case of AUL with myelofibrosis with an unusual morphology.

\section{CASE REPORT}

A 40 year female presented to the outpatient department with anaemia and hepatosplenomegaly. The hematologic
How to Cite: Roopa Urs AN, Bhavani K. Acute Undifferentiated Leukemia With Myelofibrosis - A Case Report. J Basic Clin Appl Health Sci. 2018;1:43-5 
parameters were investigated with hemoglobin $(\mathrm{Hb})$ $3.9 \mathrm{~g} / \mathrm{dl}$; platelet count is 53000 cells /cumm; and white blood cells (WBC) 2800cells/cumm having neutrophils $72 \%$, myelocyte- $2 \%$, metamyelocyte- $4 \%$ and lymphocytes- $22 \%$. On peripheral blood smear red blood cells $(\mathrm{RBC})$ showed normocytic normochromic cells with tear drop cells and 4 nucleated RBCs/100 WBCs. White blood cells showed leucopenia with neutrophilic predominance, toxic change and shift to left. Platelets were reduced in number. Retic count was $5 \%$. Thus peripheral smear was reported as pancytopenia with leucoerythroblastic blood picture and advised for bone marrow studies. Bone marrow aspirate was done and the smears were diluted and no opinion was possible. The bone marrow biopsy showed infiltration of blasts with scant cytoplasm, round to irregular nucleus, dispersed chromation.(Figure 1,2) Special stains such as reticulin stain showed grade 3 fibrosis (Figure 3) and Masson trichrome stain was negative. Immunohistochemistry (IHC) was done with a panel of markers on the bone marrow biopsy and showed CD 34 positivity (Figure 4) and negative for Tdt, MPO, CD3, CD20, CD61 markers. Erythroid and plasmacytoid cell linage markers were also negative. There was no prior hospitalization or treatment history. In view of the above test results finally diagnosed as Acute undifferentiated leukemia with myelofibrosis. There was no follow up smears as the patient was discharged but later after 9 months patient came for follow up and for further treatment with symptoms of fever on and off, weight loss and myalgia. Hemogram showed Hb:3.6gms/ dl with Normocytic normochromic red blood cells, tear drop cells, elongated cells, leucocytosis with $57 \%$ blasts, mild eosinophilia, myeloid left shift. Platelets were markedly reduced $(5,000 / \mathrm{cu} \mathrm{mm})$. Periodic Schiff stain (PAS) stain was done on the peripheral smear and it showed block positivity in the blast cells. With these follow up investigations, it was later differentiated as acute lymphoblastic leukemia.

\section{DISCUSSION}

AULs arise presumably from clonal expansion from the most primitive hematopoietic cells or from the poorly differentiated hematopoietic cells. The incidence of AUL is probably less than $0.5 \% .{ }^{6}$ Bassan et al had described about the largest study of 6 cases of AUL in 1992. ${ }^{7}$ AULs fall under the 2008 WHO classification of acute leukemia of ambiguous lineage. The leukemia classified into this category do not have a phenotype of a single cell lineage that allows for a simple clear cut classification and are therefore a diagnosis of exclusion. ${ }^{2}$
Figure1: Bone marrow biopsy showing hypocellular marrow with blasts (H\&E,x40)

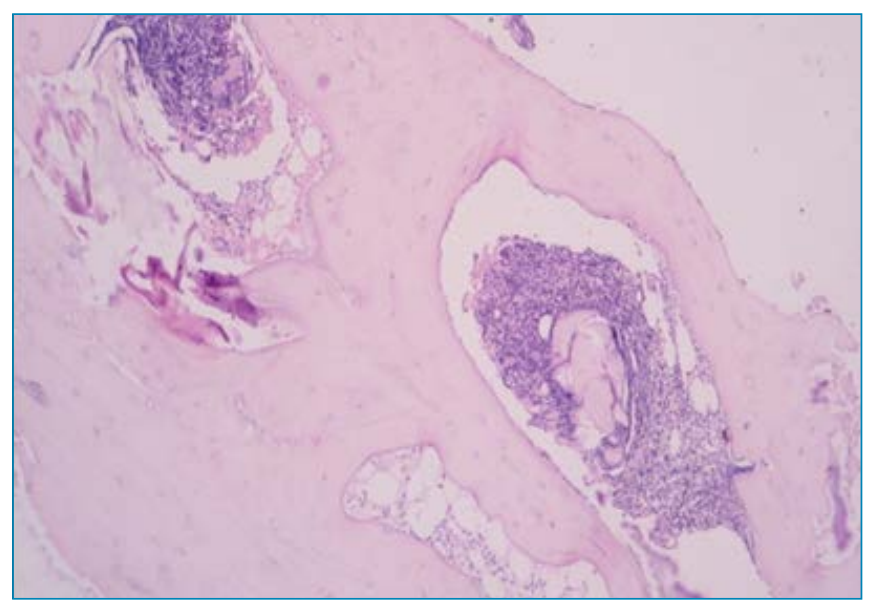

Figure 2: Bone marrow biopsy showing blasts with scant cytoplasm, round to irregular nucleus and few with prominent nucleoli (H\&E,x400)

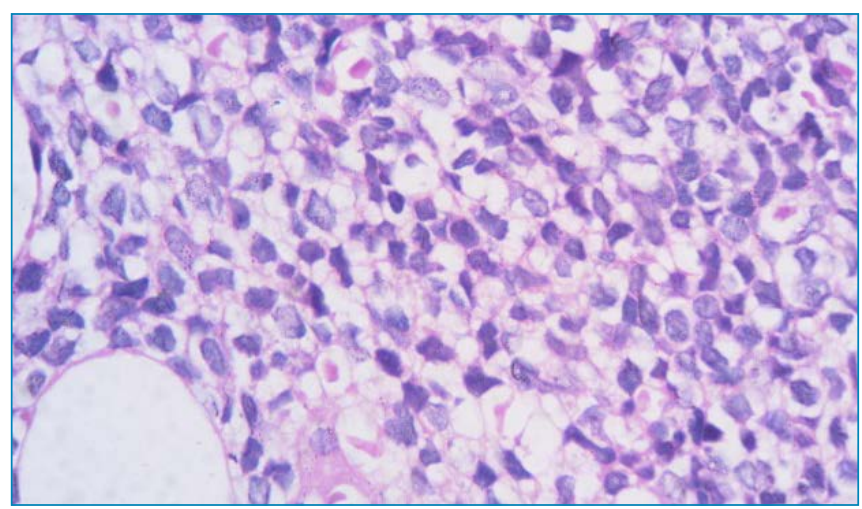

Figure 3: Reticulin stain showing grade 3 fibrosis (x400)

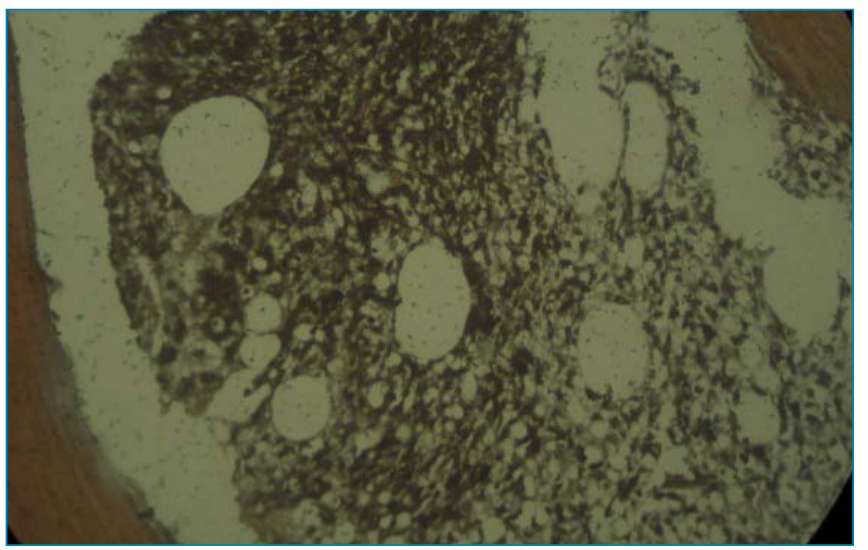

AULs typically express no more than one surface membrane antigen of any given lineage. Also, by definition they lack T-cell-specific, myeloid-specific, and B-lineage-specific markers, as well as other lineage- 
Figure 4: Postive immunohistochemical marker for CD34 (x400)

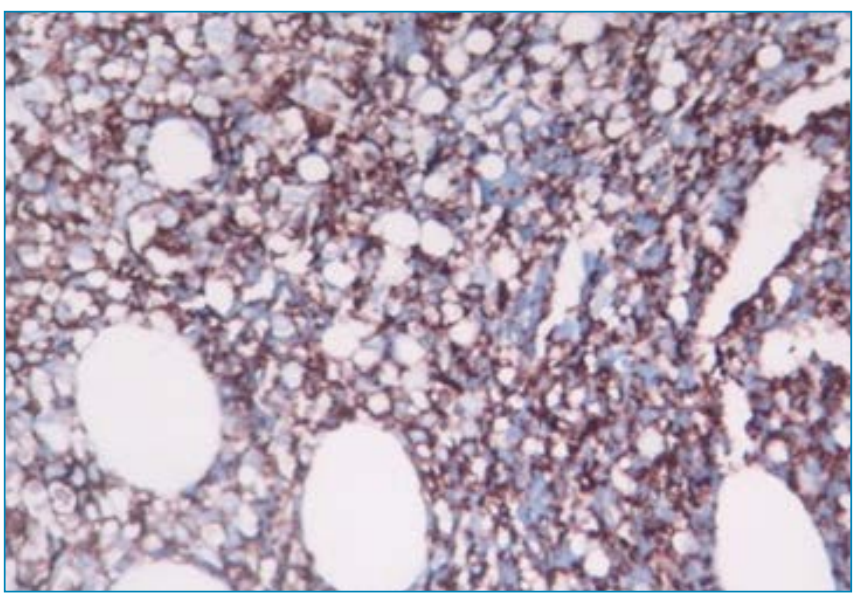

specific markers (those for plasmacytoid dendritic cells, erythroid precursors, and megakaryocytes). AULs are usually negative for MPO and the esterases by enzyme cytochemistry. The blasts often express CD34, CD38, TdT and/or HLA-DR which are all non lineage-specific markers of immaturity or activation. If none of the uncommon lineage specific markers are present, the diagnosis of AUL can be made. ${ }^{8}$ Similar features were noted in our case, in addition with features of myelofibrosis.

AULs has been differentiated in few cases in the literature. More than half of the leukemias that are morphologically undifferentiated, has shown positive reaction with myeloperoxidase which points towards early myeloid differentiation, but the immunological markers were inconclusive. Hence these leukemias has also been referred to as AML-M0. ${ }^{9}$ Also these undifferentiated tumors has shown differentiation to Langherans cell -like cells in lymph nodes. ${ }^{10}$ Our case has been differentiated to acute lymphoblastic leukemia and no literatures was found with this differentiation. Follow up AUL cases which has differntiated will be beneficient with better management options.

As AULs are rare, therefore much has not been elucidated about this hematopoietic neoplasm. Their frequency, predilection for involvement of sites other than blood and bone marrow, genetic abnormalities and progression/prognosis are not clearly known. In addition, the morphologic features of the blasts are indistinct and show no distinguishing features from blasts of other classifiable leukemias. It is important to diagnose and classify acute leukemias as it affects patient management options. Receptor studies, gene expression, and in vitro culture studies may, in the near future, contribute substantially to our knowledge about these undifferentiated blasts
The prognosis of AUL patients is generally considered as poor, although the information is too scanty to make any definitive statements. ${ }^{4} \mathrm{High}$ frequency of unfavourable cytogenetic changes, high expression of immature markers, non lineage specific antigen receptor rearrangements may be responsible for poorer prognosis.

\section{CONCLUSION}

The present case had undifferentiated features with myelofibrosis morphologically as well as by Immunohistochemistry. Very few cases have been differentiated and has been found in literature. As only few cases of AUL has been described in literature the exact significance of AUL morphology was difficult to assess. However, before categorizing leukemia as undifferentiated, it is necessary to perform immunophenotyping with a comprehensive panel of monoclonal antibodies.

\section{CONFLICTS OF INTEREST}

\section{None}

\section{References}

1. Bennett JM, Catovsky D, Daniel MT, Flandrin G, Galton DA, Gralnick HR et al. Proposals for the classification of the acute leukaemias.French-American-British (FAB) co-operative group. $\mathrm{Br}$ J Haematol. 1976;33:451-8

2. Borowitz MJ, Bene MC, Harris NL, Porwit A, Matutes E:Acute leukemias of ambiguous lineage. In: Swerdlow SH, Campo E, Harris NL, et al, eds. World Health Organization Classification of Tumours, $4^{\text {th }}$ edition. Lyon: International Agency for Research on Cancer (IARC),2008: 150-5.

3. Campana D, Hansen-Hagge TE, Matutes E, Coustan-Smith E, Yokota S, Shetty V et al. Phenotypic, genotypic, cytochemical, and ultrastructural characterization of acute undifferentiated leukemia. Leukemia. 1990;4:620-4

4. Cuneo A, Ferrant A, Michaux JL, Bosly A, Chatelain B, Stul M et al. Cytogenetic and clinicobiological features of acute leukemia with stem cell phenotype: study of nine cases. Cancer Genet Cytogenet. 1996 ;92:31-6

5. Brito-Babapulle F, Pullon H, Layton DM, Etches A, Huxtable A, Mangi $\mathrm{M}$ et al. Clinicopathological features of acute undifferentiated leukaemia with a stem cell phenotype. Br J Haematol. 1990;76:210-4

6. Shende AC, Bonagura VR, Cheah MS, Lanzkowsky P. Acute undifferentiated leukemia (AUL): a case report and a proposed system of classification. Am J Hematol 1992;40: 234-7.

7. Bassan R, Biondi A, Benvestito S, Tini ML, Abbate M, Viero P et al. Acute undifferentiated leukemia with CD7+ and CD13+ immunophenotype. Lack of molecular lineage commitment and association with poor prognostic features. Cancer. 1992;69:396-404

8. Kakimoto A, Otsubo K, Hanawa M, Kuwabara T, Futaki-Sanbe T, Saito $\mathrm{H}$ et al. Acute undifferentiated leukemia or minimally differentiated acute myeloid leukemia:Further emphasis on molecular analysis in leukemia diagnosis. Juntendo Medical J. 2016; 62: 37-41.

9. Vant veer MB. The diagnosis of acute leukemia with undifferentiated or minimally differentiated blasts. Ann hematol 1992;64:161-5

10. Fukuta T, Nakamoto S, Hashimoto Y,Tanaka T, Tokuyasu Y, Maruyama J et al. Acute undifferentiated leukemia showing differentiation to Langerhans cell like cells in lymph nodes. Rinsho Ketsueki.2017;58:26-31 